\title{
Classifier Selection Strategies for Label Fusion Using Large Atlas Databases
}

\author{
P. Aljabar ${ }^{1}$, R. Heckemann ${ }^{2}$, A. Hammers ${ }^{3}$, J.V. Hajnal ${ }^{2}$, \\ and D. Rueckert ${ }^{1}$ \\ ${ }^{1}$ Department of Computing, Imperial College London, UK \\ ${ }^{2}$ Imaging Sciences Department, MRC Clinical Sciences Centre, Imperial College \\ London, UK \\ ${ }^{3}$ Division of Neuroscience and Mental Health, MRC Clinical Sciences Centre, \\ Imperial College London, $\mathrm{UK}^{\star}$
}

\begin{abstract}
Structural segmentations of brain MRI can be generated by propagating manually labelled atlas images from a repository to a query subject and combining them. This method has been shown to be robust, consistent and increasingly accurate with increasing numbers of classifiers. It outperforms standard atlas-based segmentation but suffers, however, from problems of scale when the number of atlases is large. For a large repository and a particular query subject, using a selection strategy to identify good classifiers is one way to address problems of scale. This work presents and compares different classifier selection strategies which are applied to a group of 275 subjects with manually labelled brain MR images. We approximate an upper limit for the accuracy or overlap that can be achieved for a particular structure in a given subject and compare this with the accuracy obtained using classifier selection. The accuracy of different classifier selection strategies are also rated against the distribution of overlaps generated by random groups of classifiers.
\end{abstract}

\section{Introduction}

As increasing numbers of MR images have become available over recent years, the creation and maintenance of repositories of atlases consisting of MR images with corresponding reliable structural segmentations (manual or otherwise) has become more feasible. It has been a natural consequence to use such expert annotations to assist in providing automatic segmentations of query or unseen images. A popular approach is atlas-based segmentation, where a segmented anatomical atlas is registered with a query subject and the atlas labelling is propagated to give an estimate for the segmentation in the query subject 12 . If multiple atlases are available, the labels from each atlas can be aligned with the query image and treated as classifiers. A rule can be applied to combine or fuse these classifiers to generate a segmentation estimate. This can be a simple

\footnotetext{
* We are grateful to David Kennedy at the Centre for Morphometric analysis for providing the data for this work and we are grateful to the EPSRC who funded this work under the IBIM project.
} 
majority vote rule for each voxel [3] or a more sophisticated approach based on, for example assigning weights to classifiers [4.

Using a database of images of bee brains, Rohlfing et al. [3] showed that fusing the classifiers according to a simple vote or majority rule is robust and accurate compared with other methods. The vote rule has also been shown to outperform other classifier combination methods for more general applications [5]. In the context of the segmentation of human brain structures, Heckemann et al. [6] showed that this approach exceeds the accuracy of previous automatic methods and compares well with manual labelling. Using 30 expert labellings of human brains [7], a model was developed for the asymptotic level of accuracy achievable as the number of input classifiers increases. For classifier numbers beyond 15 to 20 atlases, the improvement in terms of segmentation accuracy tended to be very small.

If such an approach is applied to a repository consisting of a very large number of atlases, other factors become relevant. For example, the effort required to segment an unseen subject increases linearly according to the number of subjects if every atlas is registered to the query image. It is also likely that the population represented among the atlases is diverse with respect to factors such as age or pathology. This means that certain subjects in the database are more appropriate than others as potential classifiers for a query image. In the context of these considerations, and the diminishing returns from the use of increasing number classifiers, it is desirable to have a strategy for selecting atlases from among the population that are the most appropriate for the particular query subject and for the structure(s) which need to be segmented. Wu et al. investigated methods for optimal selection of a single template for atlas-based segmentation [8]. Our work contrasts with this in that we select multiple atlases for subsequent fusion.

In this work we present an investigation of practical selection strategies using a database consisting of $275 \mathrm{MR}$ images and accompanying manual labels. We rate the performance of selections against an estimate of the 'best' possible segmentation accuracy and against the performance of random groups of classifiers. Finally we investigate the effect of selecting increasing numbers of classifiers from a ranked set of atlases.

\section{Data and Methodology}

The data used consisted of T1 weighted MR brain images of 275 male and female subjects with ages between 4 and 83 years acquired in multiple centres. Various structures in these images were manually delineated by the Centre for Morphometric Analysis (Massachusetts General Hospital, Charlestown, MA) following a single segmentation protocol. Manual delineations for 35 structures were present in all images. For our experiments, a number of subjects were randomly selected and used as test or query subjects and the remaining subjects' images and manual segmentations were treated as an atlas repository.

In this work, the term 'atlas' describes the pairing of an anatomical image and its corresponding manual labelling. If the anatomies of an atlas and a query 
subject are in alignment, the atlas labels can be considered to be a 'classifier', providing an estimate for a segmentation of the query. If multiple atlases are available as classifiers for a particular query, then these classifiers can be 'fused' or 'combined' to provide an improved segmentation estimate [36]. We describe as a fusion experiment the process of selecting a number of the repository subjects as classifiers and subsequently combining them with the majority vote rule to provide a fused segmentation estimate for a particular structure within a chosen query subject.

All subjects were registered to the MNI Brainweb single subject simulated T1 weighted MR image [9] to image avoid the computational burden that would be associated with registering every atlas to every query subject. The registrations consisted of an affine registration step followed by a non-rigid registration using a B-spline based free form deformation model [10] which was applied hierarchically with successive control point spacings of 20,10 and $5 \mathrm{~mm}$. The metric used during registration was normalised mutual information (NMI) [11. The resulting transformations were used to spatially normalise all anatomical images and their manual labels. After spatial normalisation, a region of interest (ROI) was defined for each structure studied as the minimum bounding box containing the structure for all repository subjects expanded by two voxels along each dimension.

The strategy for selecting atlases as classifiers is the main focus of this work, and this was carried out based on a set of ranks assigned to the repository images. The following describes the different methods used to select atlases from the repository:

- Classifier selection based on segmentation similarity: For a given query subject, the Dice overlaps 12 between the subject's manual label and all the repository subjects were found. The repository images were then ranked according to these values. Clearly, this method is not a realistic option in a practical setting as the manual segmentations for query subjects are generally unavailable (in fact, the segmentation of the query subjects is the overall goal). The accuracy of the resulting fused segmentation does, however, provide a reference value to compare against other selection methods.

- Classifier selection based on image similarity: For a given query subject, a number of different image similarity metrics can be calculated using the T1 weighted images of the query and of the repository atlases. The similarity ranking strategy breaks down into four methods based on the metrics used: sum of squared differences (SSD), cross correlation (CC), mutual information (MI) and normalised mutual information (NMI). The metric was calculated using voxels in the ROI defined for the chosen structure. The repository atlases were then ranked according to the resulting similarity metric values.

- Classifier selection based on demographics: In general, it is possible to select classifiers based on non-image information, e.g. age, sex or pathology. We have selected from atlases that were ranked according to how close their ages were to the query subject's age. This ranking is therefore independent of the image data. 


\section{$3 \quad$ Experiments and Results}

\subsection{Experiments}

Fifteen classifiers were used for each fusion experiment and the accuracy of each resulting segmentation was measured by its Dice overlap with the manual segmentation for the query subject, which was treated as the 'gold standard'. To assess the performance of a selection method, the overlap of the resulting segmentation with the gold standard label was calculated and compared with the distribution of overlaps obtained from multiple fusions of randomly selected classifiers $(N=1000,15$ classifiers per experiment). In this work, we present the results of experiments using the hippocampus, the lateral ventricles and the thalamus which were chosen as representative structures in terms, size, image intensity and of the segmentation accuracy achievable by automated methods. In previous work [6], average Dice coefficients for the accuracy of fusion based segmentations of these structures have been 0.90 for the lateral ventricles and the thalamus and 0.82 for the hippocampus. Each structure's left-right pair was treated as a single structure.

A number of subjects were used as a test set and classifiers were selected from the remaining subjects which were used as a repository. For selection based on segmentation or image similarity, 20 subjects were randomly selected as the test set. For selection based on age proximity, the test set consisted of 15 random subjects equally divided among each of three age intervals: $0-10,30-45$ and $70+$ years.

The effect of selecting different numbers of ranked classifiers was also investigated. For each of the 20 subjects in the test set used for similarity selection, the repository atlases were ranked by segmentation similarity. Subsequently, starting with the top-ranked atlas, successively larger numbers of ordered atlases were used as classifiers, i.e. each group of classifiers was formed from the previous group with the inclusion of one further classifier.

\subsection{Selection Strategy Comparisons}

After fusion of 1000 sets of 15 random classifiers for all 20 query subjects, the mean (SD) overlap values were: Hippocampus 0.79 (0.03), lateral ventricle 0.90 (0.02), thalamus $0.88(0.02)$. The random overlap distributions obtained for the hippocampus and lateral ventricle are shown in Figure 1 as box-and-whisker plots. Each box shows the lower quartile, the median and the upper quartile $\left(q_{1}, q_{2}\right.$ and $\left.q_{3}\right)$. The whisker lengths are set at 1.5 times the inter-quartile range (distributions for the thalamus are qualitatively similar but were omitted for reasons of space). A negligible number of overlap values fell outside the whisker ranges which were not plotted for readability: $0.2 \%$ gave values above the whiskers and $2 \%$ below. Figure 1 also shows the overlaps achieved after similarity based selection, these can be compared against the random overlap distributions.

To summarise the performance of the image similarity selection methods, their overlap measures with the gold standard were compared with the quartiles 

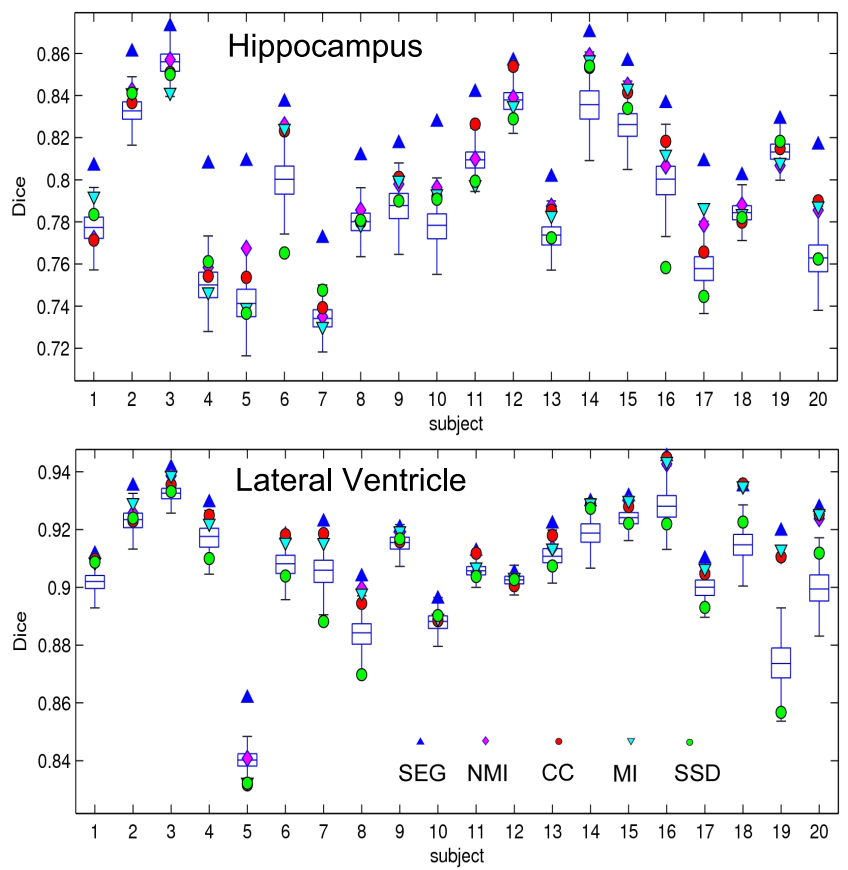

Fig. 1. Comparisons of performance for the segmentation similarity (SEG) and image similarity selection methods for 20 randomly selected test subjects. The similarity measures used were normalised mutual information (NMI), mutual information (MI), cross-correlation (CC), and sums of squared differences (SSD). For comparison, each box-and-whisker plot shows the Dice overlap distribution for 1000 random fusions of 15 classifiers.

Table 1. Performance ratings for image similarity selection using quartiles and z-scores. $d$ represents the Dice overlap obtained by a particular metric, $q_{1}, q_{2}$, $q_{3}$ : the quartiles for the random overlap distribution. Entries indicate the number of each case among the 20 query subjects. Bottom row: Mean z-scores for each combination of similarity metric and structure across all query subjects.

\begin{tabular}{|c|c|c|c|c|c|c|c|c|c|c|c|c|}
\hline & \multicolumn{4}{|c|}{ Lateral Ventricle } & \multicolumn{4}{|c|}{\begin{tabular}{|l|} 
Hippocampus \\
\end{tabular}} & \multicolumn{4}{|c|}{ Thalamus } \\
\hline & NMI & $\mathrm{MI}$ & $\mathrm{CC}$ & SSD & NMI & MI & $\mathrm{CC}$ & SSD & NMI & $\mathrm{MI}$ & $\mathrm{CC}$ & SSD \\
\hline$q_{3} \leq d \leq 1$ & 16 & 16 & 15 & 5 & 14 & 11 & 13 & 8 & 16 & 19 & 17 & 2 \\
\hline$q_{2} \leq d<q_{3}$ & 2 & 3 & 2 & 4 & 4 & 1 & 3 & 2 & 4 & 0 & 2 & 5 \\
\hline$q_{1} \leq d<q_{2}$ & 2 & 0 & 1 & 1 & 1 & 6 & 1 & 4 & 0 & 1 & 1 & 7 \\
\hline $0 \leq d<q_{1}$ & 0 & 1 & 2 & 10 & 1 & 2 & 3 & 6 & 0 & 0 & 0 & 6 \\
\hline Mean z & 1.8 & 1.8 & 1.7 & $\overline{-0.4}$ & 1.2 & 0.8 & 1.2 & $\overline{-0.1}$ & 2.1 & 1.9 & 2.0 & -0.5 \\
\hline
\end{tabular}

of the random overlap distributions. The quartiles define four intervals $\left[0, q_{1}\right)$, $\left[q_{1}, q_{2}\right),\left[q_{2}, q_{3}\right),\left[q_{3}, 1\right]$ and the number of overlaps within each interval for each image similarity selection method and structure are shown in Table 1 With 


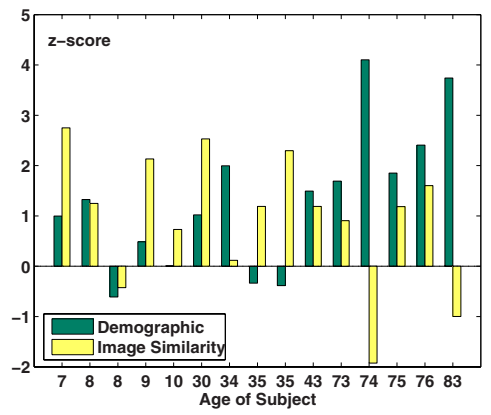

Fig. 2. A comparison of age based selection (green) with image similarity selection (NMI, yellow) using hippocampus overlap z-scores for subjects with different ages. The subjects' ages are on the horizontal axis.
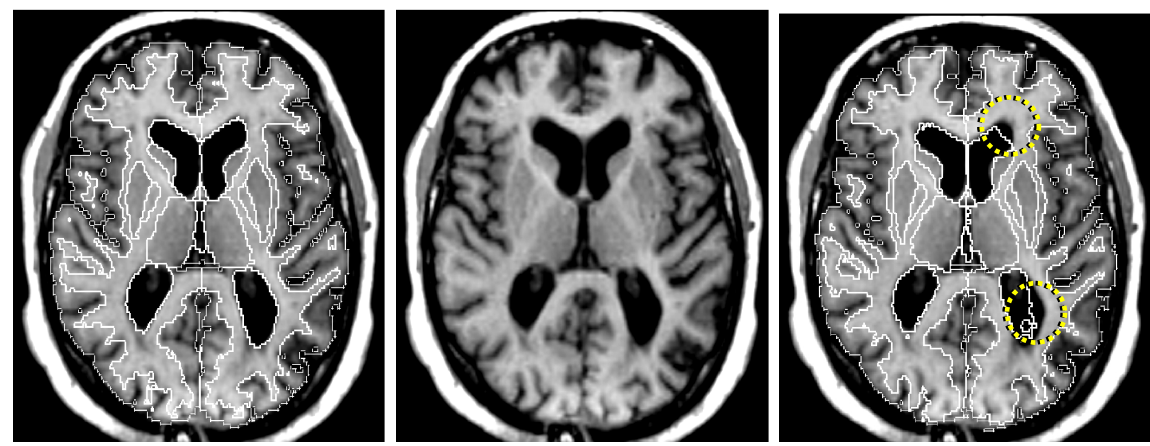

Fig. 3. Label fusion for the image of a 74 year old query subject. Left: Segmentation obtained by fusing the nearest 15 subjects in age. Centre: T1 weighted image. Right: A segmentation based on 15 randomly selected classifiers; dashed circles indicate regions of segmentation failure.

the exception of SSD, the image similarity selection methods all outperformed the median random overlap in 17 or more cases for the lateral ventricle. NMI selection exceeded the median in 18 of the 20 subjects for the hippocampus and lateral ventricle and in all cases for the thalamus.

The overlap values for each ranking method were also rated by converting them to z-scores using the mean and standard deviation of the random overlap distribution for the corresponding query subject and structure. The z-score expresses the signed difference between the measured value and the distribution mean as a multiple of standard deviations. The mean z-scores for the image similarity metrics are shown in Table 1] where it can be seen that the highest mean z-score was always achieved by image similarity selection using NMI. The mean z-scores after segmentation similarity selection were 3.2, 4.5 and 3.6 for the lateral ventricle, hippocampus and thalamus.

Using the test set for age based selection, classifiers were selected for each query subject according to age proximity and overlaps were calculated for the resulting 


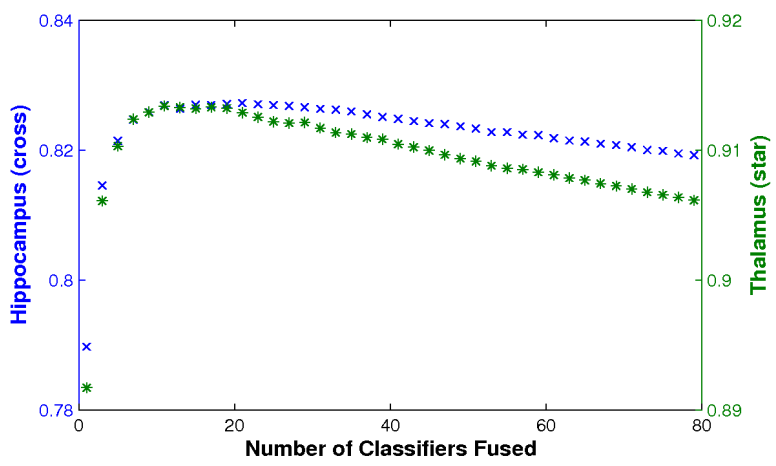

Fig. 4. Selecting increasing numbers of classifiers from the repository after after segmentation similarity ordering: The effect upon thalamus and hippocampus overlaps

fusions. Figure 2 shows these overlaps in the form of z-scores where the figures obtained after image similarity (NMI) selection for the same subjects are also shown for comparison. As an illustration, the image for a particular 74 year old subject is shown in Figure 3. This diagram also shows the labels produced by fusing the 15 age-selected classifiers along with a segmentation produced using 15 randomly selected classifiers. The fusion of randomly selected classifiers has, for example, overestimated the white matter on the right side of the brain and the segmentation of the right ventricle has failed in this case.

\subsection{Varying the Number of Classifiers}

In separate experiments, using the similarity selection test set, increasing numbers of classifiers were selected from an ordered set of atlases and fused. The atlases were ordered by segmentation similarity for each structure. The mean overlaps obtained across the test subjects for the hippocampus and thalamus are shown in Figure 4 (The data for the ventricles are similar but have been omitted for reasons of space). The main feature of these results is that the overlap values improve rapidly, reach their highest value for approximately 10-20 classifiers and subsequently decrease gradually as more classifiers are chosen from the ranked set.

\section{Discussion}

We have presented and tested strategies for classifier selection which can reduce the effects of scale when using large atlas repositories to segment structures in unseen images. The tested selection strategies are based on segmentation or image similarity and on age. Performance of the different methods was assessed in comparison with the distributions of overlap values obtained from a series of random classifier fusion experiments. In order to avoid the computational burden of registering every atlas with every query subject, the selection of classifiers and 
their fusion was carried out in a single reference space. We are currently adapting these methods to use reference space classifier selection in order to generate further improvements in segmentation accuracy in the query subject's native space. The high overlaps achieved by segmentation similarity based selection suggest they are good upper bound estimates for the accuracy possible with classifier fusion.

Using image similarity selection, the performance figures clearly show SSD as being the least reliable in identifying a good set of classifiers. The other metrics, NMI, MI and CC, vary more subtly; NMI selection generally performed best, being most likely to achieve an overlap value above the median of the random distribution and obtaining the highest z-scores throughout. This may be expected since NMI was used as the metric during registration.

How well a structure can be segmented for each query subject is estimated by the overlap distributions of randomly fused labels. Predictably, these show that the lateral ventricles are the easiest to segment. Additionally, the random overlap averages vary significantly between subjects showing a subject dependence for the segmentation accuracy. It is also noted that the quality of fused segmentations for one structure does not directly translate to another. For example subject 19 (Figure 1) has overlaps that are good for the hippocampus but poor for the lateral ventricle relative to the group. This provides some justification for the use of regional local metrics for selection.

Using age-based selection, the z-scores for NMI-based and age-based selection (Figure 2) suggest comparable performance for the young and middle aged groups. For the older group, however, selection by age significantly outperformed NMI selection in every case suggesting the potential demographic criteria have for increasing segmentation accuracy through classifier selection. The segmentations for the 74 year old subject shown in Figure 3 highlight how random classifiers can fail where age-based atlas selection provides a much better estimate. In our experience, such segmentation failures are possible for subjects with, for example, enlarged ventricles when using normal atlases. Selection strategies that combine image similarity and demographic data should provide an interesting area for further work.

The selection of increasing numbers of ranked classifiers after ordering the repository images $(N=255)$ showed that the highest overlap values were achieved for groups of 10-20 classifiers. This indicates that the choice of 15 subjects for the fusion experiments was reasonable. Moreover, the gradual decline in overlap values as more of the ordered classifiers are fused suggests that there is merit in avoiding the use of large numbers of classifiers when the repository is large and selection is applied. Clearly as the number of classifiers continues to increase, the resulting fused segmentations will approach the population mean and the overlaps will reach an asymptotic value. Heckemann et al. 6] showed that selecting increasing numbers of random sets of classifiers gave overlap values that monotonically increased to an asymptotic value, and they presented a model for convergence to this value. We feel that the limiting overlap value 
obtained by selecting from an ordered set of classifiers will be the same, and establishing this could prove an interesting task for further work.

In conclusion, we have presented and compared different strategies for classifier selection that should help towards reducing the computational burden associated with the use of large atlas databases. The results suggest that image and demographic based classifier selection can perform well in terms of segmentation accuracy and that there is merit in ranking and limiting the number of classifiers used when a large number are available.

\section{References}

1. Svarer, C., Madsen, K., Hasselbalch, S.G., Pinborg, L.H., Haugbol, S., Frokjaer, V.G., Holm, S., Paulson, O.B., Knudsen, G.M.: MR-based automatic delineation of volumes of interest in human brain PET images using probability maps. NeuroImage 24, 969-979 (2005)

2. Iosifescu, D., Shenton, M., Warfield, S., Kikinis, R., Dengler, J., Jolesz, F., Mccarley, R.: An automated registration algorithm for measuring MRI subcortical brain structures. Neuroimage 6, 13-25 (1997)

3. Rohlfing, T., Brandt, R., Menzel, R., Maurer Jr., C.R.: Evaluation of atlas selection strategies for atlas-based image segmentation with application to confocal microscopy images of bee brains. NeuroImage 21, 1428-1442 (2004)

4. Warfield, S., Zou, K., Wells, W.: Simultaneous truth and performance level estimation (STAPLE): an algorithm for the validation of image segmentation. IEEE Transactions on Medical Imaging 23, 903-921 (2004)

5. Kittler, J., Hatef, M., Duin, R., Matas, J.: On combining classifiers. IEEE Transactions on Pattern Analysis and Machine Intelligence 20, 226-239 (1998)

6. Heckemann, R.A., Hajnal, J.V., Aljabar, P., Rueckert, D., Hammers, A.: Automatic anatomical brain MRI segmentation combining label propagation and decision fusion. Neuroimage 33, 115-126 (2006)

7. Hammers, A., Allom, R., Koepp, M., Free, S., Myers, R., Lemieux, L., Mitchell, T., Brooks, D., Duncan, J.: Three-dimensional maximum probability atlas of the human brain, with particular reference to the temporal lobe. Human Brain Mapping 19, 224-247 (2003)

8. Wu, M., Rosano, C., Lopez-Garcia, P., Carter, C.S., Aizenstein, H.J.: Optimum template selection for atlas-based segmentation. NeuroImage 34, 1612-1618 (2007)

9. Cocosco, C., Kollokian, V., Kwan, R.S., Evans, A.: BrainWeb: Online interface to a 3D MRI simulated brain database. NeuroImage 5 (1997)

10. Rueckert, D., Sonoda, L., Hayes, C., Hill, D., Leach, M., Hawkes, D.: Non-rigid registration using free-form deformations: Application to breast MR images. IEEE Transactions on Medical Imaging 18, 712-721 (1999)

11. Studholme, C., Hill, D.L.G., Hawkes, D.J.: An overlap invariant entropy measure of 3D medical image alignment. Pattern Recognition 32, 71-86 (1998)

12. Dice, L.R.: Measures of the amount of ecologic association between species. Ecology 26, 297 (1945) 